\title{
Dosis letal media y reducción media del crecimiento por radiación gamma en pasto africano (Eragrostis lehmanniana Ness)
}

\section{Median lethal dose and average growth reduction due to gamma radiation in African grass (Eragrostis lehmanniana Ness)}

\author{
Alan Álvarez-Holguín ${ }^{1}$, Carlos Raúl Morales-Nieto ${ }^{1 *}$, Carlos Hugo Avendaño-Arrazate ${ }^{2}$, Eduardo \\ Santellano-Estrada ${ }^{1}$, Alicia Melgoza-Castillo ${ }^{1}$, M. Eduviges Burrola-Barraza ${ }^{1}$, Raúl Corrales-Lerma ${ }^{1}$ \\ ${ }^{1}$ Facultad de Zootecnia y Ecología. Universidad Autónoma de Chihuahua. Perif. Francisco R. Almada, Km 1. CP. 31000 \\ Chihuahua, Chihuahua, México. \\ ${ }^{2}$ Instituto del Nacional de Investigaciones Forestales, Agrícolas y Pecuarias (INIFAP). Campo Experimental Rosario Izapa, \\ Carretera Tapachula - Cacahoatán kilómetro 18. Código postal 30870. Tuxtla Chico, Chiapas. México. \\ *Autor de correspondencia: cnieto@uach.mx
}

Nota científica recibida: 08 de noviembre de 2016 aceptada: 09 de agosto de 2017

RESUMEN. El objetivo fue determinar la dosis letal media $\left(\mathrm{DL}_{50}\right)$ y la reducción media del crecimiento $\left(\mathrm{RG}_{50}\right)$ en pasto africano (Eragrostis lehmanniana) para determinar la dosis óptima de irradiación gamma para inducir mutagénesis en esta especie. Las variables evaluadas fueron porcentaje de germinación, índice de velocidad de germinación, longitud de plúmula, longitud de radícula, rendimiento de forraje, producción de semilla, número de tallos, altura de planta, diámetro de macollo e índice de concentración de clorofila. Se realizó un análisis de tendencia para cada variable y con la ecuación de regresión resultante se estimó la $\mathrm{DL}_{50}$ y $\mathrm{RG}_{50}$. Los resultados obtenidos indican que la $\mathrm{DL}_{50}$ se encontró a $2076 \mathrm{~Gy}$, mientras que la $\mathrm{RG}_{50}$ fluctuó entre 1357 y $1900 \mathrm{~Gy}$. Para el mejoramiento genético de esta especie se recomienda utilizar la media ponderada de 1533 Gy de ambos parámetros.

Palabras clave: Germinación, mutagénesis, radiosensibilidad, rayos gamma

ABSTRACT. The objective was to determine the median lethal dose $\left(\mathrm{LD}_{50}\right)$ and average growth reduction $\left(G \mathrm{R}_{50}\right)$ in African grass (Eragrostis lehmanniana) to determine the optimal dose of gamma irradiation to induce mutagenesis in this species. The variables evaluated were germination percentage, germination speed index, plumule length, radicle length, forage yield, seed production, number of stems, plant height, tiller diameter and chlorophyll concentration index. A trend analysis was performed for each variable and with the resulting regression equation the $\mathrm{LD}_{50}$ and $\mathrm{GR}_{50}$ were estimated. The results obtained indicate that the $\mathrm{LD}_{50}$ was found at $2076 \mathrm{~Gy}$, while the $\mathrm{GR}_{50}$ fluctuated between 1357 and $1900 \mathrm{~Gy}$. For the genetic improvement of this species, it is recommended to use the weighted average of $1533 \mathrm{~Gy}$ of both parameters.

Key words: Germination, mutagenesis, radiosensitivity, gamma rays.

\section{INTRODUCCIÓN}

La radiación gamma es un agente mutagénico que se utiliza para inducir variabilidad genética en programas de mejoramiento vegetal. Antes de iniciar un programa de mejoramiento genético por mutagénesis, es necesario establecer la dosis de radiación con mayor probabilidad de producir mutaciones efectivas (Ramchander et al. 2015, Ra- jarajan et al. 2016). Diversos investigadores concuerdan que la dosis donde muere el $50 \%\left(\mathrm{DL}_{50}\right)$ de los individuos irradiados, es donde existe mayor probabilidad de producir mutaciones útiles en un programa de mejoramiento genético (Golubinova y Gecheff 2011, Ángeles-Espino 2013). Mientras que otros investigadores señalan que otra dosis con alta probabilidad de producir mutaciones efectivas es donde se reduce el crecimiento en $50 \%\left(R_{50}\right)$ 
(Akgüm y Tosun 2004, Khalil et al. 2014). Lo que se debe a que con dosis bajas de radiación los impactos en el genoma son mínimos, por lo tanto los cambios fenotípicos también serán escasos. Por otro lado, con dosis altas el genoma sufre múltiples impactos que regularmente producen aberraciones o cambios negativos (Songsri et al. 2011, Thole et al. 2011).

El pasto africano (Eragrostis lehmanniana) es una especie invasora que afecta los pastizales del norte de México y se caracteriza por ser de alta prolificidad, capacidad de establecimiento y baja apetencia para el ganado (Flanders et al. 2006). Su hábito de crecimiento y rusticidad proveen al pasto africano de alta capacidad para invadir y dominar la composición de gramíneas en los pastizales donde se encuentra (Hupy et al. 2004). Sin embargo, estas características también convierten al pasto africano en una especie atractiva para rehabilitar pastizales con alto grado de degradación, donde otras especies no logran establecerse (McGlone y Huenneke 2004). Antes de utilizar esta especie es importante que se someta a un programa de mejoramiento genético para aumentar su aceptación por el ganado y disminuir su capacidad invasora (O'Reagain y Mentis 1989). No obstante, por ser una especie introducida en México, las poblaciones de pasto africano pudieran presentar una reducida variabilidad genética; por lo que la mutagénesis es una alternativa que puede generar variabilidad genética para el mejoramiento del pasto africano. Pero se desconoce la dosis con mayor probabilidad para producir mutaciones efectivas. Por lo que, el objetivo fue determinar la dosis letal media y la reducción media del crecimiento para determinar la dosis óptima de irradiación gamma e inducir mutagénesis en pasto africano.

\section{MATERIALES Y MÉTODOS}

El experimento se realizó en laboratorio e invernadero. Se evaluaron 10 dosis de irradiación en semilla de pasto africano, las cuales consistieron en la aplicación de 0 (tratamiento testigo), 100, 200, 300, 450, 600, 900, 1400, 2000 y 4000 Gray
(Gy). La irradiación se realizó en el Complejo MOSCAMED de SENASICA-SAGARPA en Metapa de Domínguez, Chiapas, México, en colaboración con el Campo Experimental Rosario Izapa, Chiapas del INIFAP. Las dosis se aplicaron en un irradiador panorámico Gamma Beam 127 MDS Nordion, con fuente de almacenamiento de $50 \mathrm{~g}$ de cobalto 60 (Co60) en seco.

\section{Prueba de germinación}

Se utilizaron cajas Petri de $90 \mathrm{~mm}$ de diámetro provistas de algodón y papel filtro. La prueba de germinación se realizó bajo un diseño experimental completamente al azar, donde la unidad experimental fue la caja Petri con 50 semillas, con cuatro repeticiones por dosis de radiación. Cada repetición fue humectada con $25 \mathrm{ml}$ de agua al inicio de la prueba y posteriormente, se suministraron riegos por aspersión de $2.0 \mathrm{ml}$ cada dos días, durante los 17 d que duró la prueba. Las cajas Petri se colocaron a temperatura ambiente de 28 $\pm 2{ }^{\circ} \mathrm{C}$. Se consideró semilla germinada aquella que alcanzó $0.5 \mathrm{~cm}$ de plúmula o de radícula. Las variables evaluadas fueron porcentaje germinación $(P G)$, índice de velocidad de germinación (Vge), longitud de plúmula (LP) y longitud de radícula (LR). Para obtener LR y LP se dejaron crecer tres plántulas por caja Petri durante siete días después de germinadas. La Vge se calculó mediante la formula propuesta por Maguire (1962):

$$
V g e=\sum \frac{n_{i}}{t}
$$

Dónde: $\quad V g e=$ velocidad de germinación, $\mathrm{n}_{i}=$ número de semillas germinadas por día, $\mathrm{t}=$ día de la germinación.

\section{Evaluación en invernadero}

Para la prueba en invernadero se utilizaron las mismas dosis de irradiación que en la prueba de germinación, con excepción de la dosis de $\mathbf{4 0 0 0}$ Gy. La cual se excluyó del análisis en invernadero por no presentar germinación. La prueba se realizó bajo un diseño experimental en bloques al azar con 10 repeticiones por tratamiento. Se sembraron 
20 semillas por maceta para asegurar emergencia y establecimiento y dejar un individuo (planta) por maceta. La siembra se realizó en bolsas de polietileno negro de $26 \mathrm{~cm}$ de altura por $18 \mathrm{~cm}$ de diámetro, perforadas en la parte inferior. Las bolsas se llenaron con $23 \mathrm{~cm}$ de suelo franco-arenoso de origen aluvial. Los riegos se suministraron hasta punto de saturación cada dos o tres días, según el desecamiento del suelo. La prueba se realizó en una sola fase de crecimiento durante 16 semanas de mayo a agosto de 2016, periodo en que la plantas alcanzaron la madurez. Las variables evaluadas fueron rendimiento de forraje (RF), producción de semilla (PS), numero de tallos (NT), altura de planta (AP), diámetro de macollo (DM) e índice de concentración de clorofila (ICC). Para el PS se recolectó la totalidad de la semilla de cada planta y se colocó en bolsas de papel. Para el RF la biomasa se cortó a $5 \mathrm{~cm}$ del suelo y se colocó en bolsas de papel. Las muestras extraídas de forraje y semilla se secaron en estufa a $65{ }^{\circ} \mathrm{C}$ durante $72 \mathrm{~h}$. Una vez secas las muestras se pesaron en balanza analítica marca Viper BC, Mettler. La AP se midió desde la base hasta el punto vegetal y el NT se contabilizó en la base de la planta. El ICC se midió en la parte media de cinco hojas seleccionadas al azar con medidor marca Opti-Sciences, modelo CCM-200.

\section{Análisis estadístico}

Los datos se analizaron mediante análisis de tendencia para cada variable y se seleccionó el modelo de regresión que presentó mayor nivel de significancia. Con la ecuación de regresión resultante se estimó la $\mathrm{DL}_{50}$ con base en el PG. La RG $\mathrm{R}_{50}$ se determinó en las variables Vge, LP, LR, RF, PS, $\mathrm{NT}, \mathrm{AP}, \mathrm{DM}$ e ICC. Los datos se analizaron mediante comparación de medias con la prueba de Dunnett a un $\alpha=0.01$, donde el factor de influencia fue la dosis de radiación. Los análisis se realizaron con el procedimiento GLM del paquete estadístico SAS 9.1.3 (2006). Con los resultados de la $\mathrm{DL}_{50}$ y $\mathrm{RG}_{50}$ obtenidos de las 10 variables evaluadas, por medio de regresión, se obtuvo la media ponderada. La $\mathrm{DL}_{50}$ se ponderó con $30 \%$, mientras que el $70 \%$ restante se dividió equitativamente entre los valores obtenidos de $\mathrm{RG}_{50}$ de las nueve variables. Cabe señalar que la mayor ponderación se le dio a la germinación, ya que la muerte del individuo claramente es el máximo daño que puede producir la radiación.

\section{RESULTADOS Y DISCUSIÓN}

Al aumentar la dosis de irradiación, el comportamiento del PG mostró una tendencia lineal negativa ( $p<0.0001)$. No obstante, las únicas dosis que presentaron diferencias $(p<0.01)$ con respecto al tratamiento testigo ( $\mathrm{T}-0$ ) fueron 1400 , 2000 y 4000 Gy. En términos generales el PG tendió a disminuir conforme aumentó la dosis de irradiación (Figura 1a). Este resultado concuerda con diversos autores, quienes encontraron que la germinación de diferentes especies de gramíneas tiende a disminuir a medida que aumenta la dosis de radiación (Golubinova y Gecheff 2011, Rajarajan et al. 2016). Esto se debe a que la radiación ionizante puede deprimir o inhibir funciones vitales de las células, lo que provoca la muerte de algunas células e incluso la muerte del embrión. Este fenómeno tiende a aumentar con el incremento de la dosis de irradiación, lo que causa disminución de la germinación (Olasupo et al. 2016). La ecuación de regresión indica que la $\mathrm{DL}_{50}$ en pasto africano se presentó a 2076.1 Gy.

Una de las gramíneas más estudias en cuestión de radiosensibilidad es el arroz (Oryza sativa). En esta especie Harding et al. (2012) al exponer a 13 variedades a radiación gamma encontraron que la $\mathrm{DL}_{50}$ varió entre 345 y $423 \mathrm{~Gy}$. Otra especie gramínea donde se ha estudiado la radiosensibilidad es mijo perla (Pennisetum glaucum), donde la $\mathrm{DL}_{50}$ fue determinada en 669 Gy (Ousmane et al. 2013). Con respecto a gramíneas forrajeras la $\mathrm{DL}_{50}$ en pasto sudan (Sorghum sudanense) varia entre 307 y $342 \mathrm{~Gy}$ para diferentes variedades (Golubinova y Gecheff 2011). No obstante, los resultados anteriores son inferiores a la $\mathrm{DL}_{50}$ obtenida en este estudio para el pasto africano. Esto probablemente se debe a que la semilla de pasto guarda bajo contenido de humedad, ya que la radiación ionizante puede provocar ruptura de enlaces cova- 

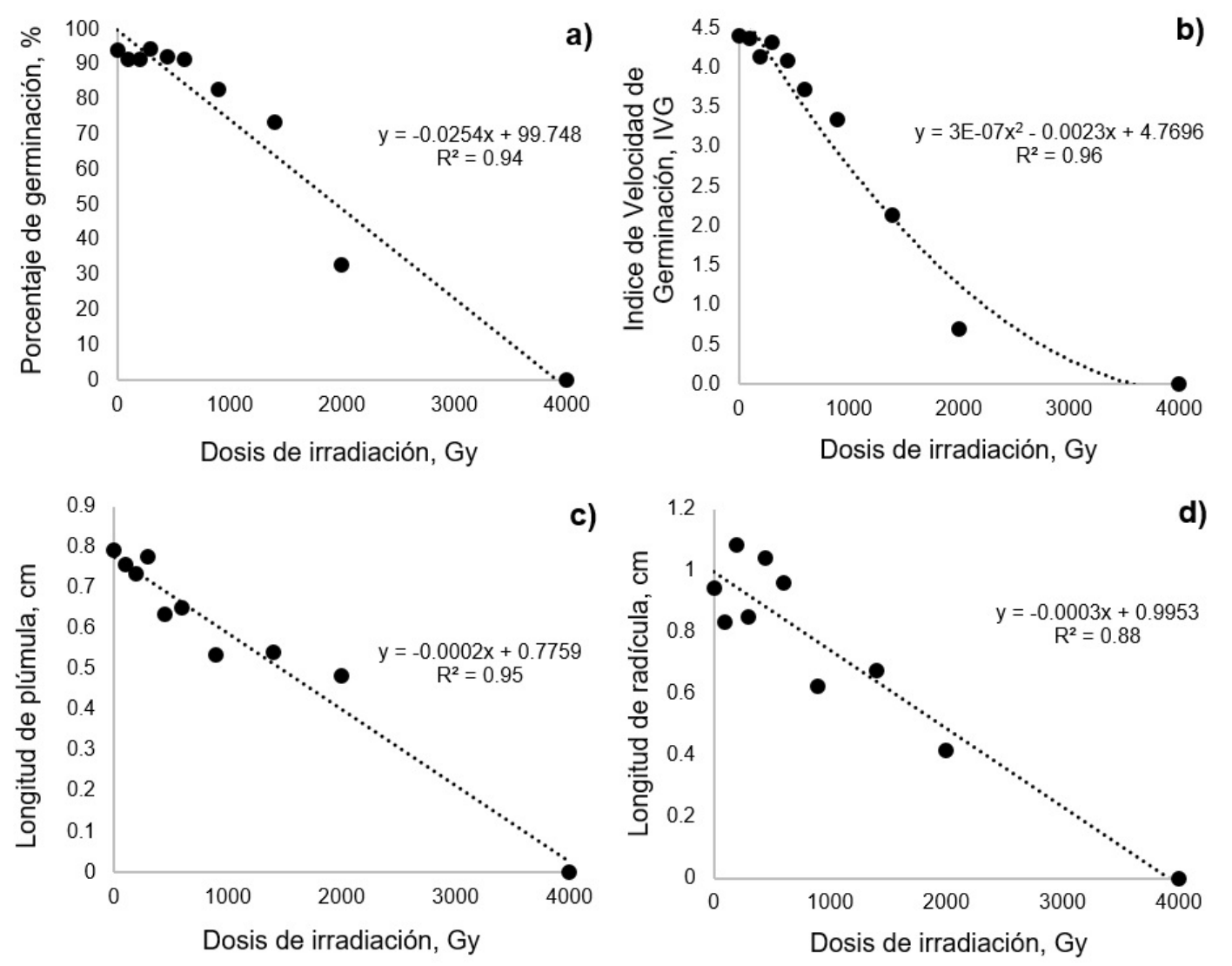

Figura 1. Efecto de diferentes dosis de irradiación con $\mathrm{CO}^{60}$ sobre el porcentaje de germinación (a), índice de velocidad de germinación (b), longitud de plúmula (c) y longitud de radícula (d) en pasto africano (Eragrostis lehmanniana).

lentes y descomposición de moléculas de agua. Lo cual forma radicales libres, que de manera indirecta dañan diferentes organelos de la célula e incluso las moléculas de ADN (Alegre 2001).

La Vge mostró una tendencia cuadrática ( $p$ $<0.0001)$. Los tratamientos 100, 200, 300 y $450 \mathrm{~Gy}$ no presentaron diferencias $(\mathrm{p}>0.01)$ con el tratamiento testigo. De los $600 \mathrm{~Gy}$ en adelante, la semilla irradiada presentó menor Vge ( $p$ $<0.01$ ) con respecto al testigo (Figura $1 \mathrm{~b}$ ). Este retraso en la germinación de las semillas irradiadas probablemente se debe a que la radiación inhibe funciones vitales para la célula como la síntesis de proteínas y la actividad enzimática, lo cual retrasa la división celular y por tanto su germinación (Chandrashekar 2014, Olasupo et al. 2016). De acuerdo a la ecuación, en esta variable la $R_{50}$ se encontró a $1357.8 \mathrm{~Gy}$.

La LP presentó una tendencia lineal negativa $(p<0.0001)$. Sin embargo, la LP disminuyó de forma significativa $(p<0.01)$ a partir de los 450 Gy (Figura 1c). Según la ecuación de regresión, en esta variable, la $R_{50}$ se ubicó en 1900.5 Gy. De manera similar, la LR mostró una tendencia lineal $(p<0.0001)$. Para esta variable solamente los tratamientos con 900, 1400, 2000 y 4000 Gy tuvieron menor $(p<0.01)$ LR que el tratamiento testigo (Figura 1d). La ecuación indica que en esta variable la $R_{50}$ se presentó a $1748.3 \mathrm{~Gy}$. Los resultados de las variables anteriores, concuerdan con lo reportado por Ambavane et al. (2014), quienes encontraron que en finger millet (Eleusine coracana) 
la LP y la LR se vieron afectadas por el incremento en la dosis de radiación. En este estudio se determinó la $\mathrm{RG}_{50}$ en $500 \mathrm{~Gy}$. Este mismo efecto fue encontrado en arroz, donde la $R_{50}$ se registró en 250 y 450 Gy para ambas variables, respectivamente (Talebi y Talebi 2012). La disminución en la elongación de la plúmula y radícula puede deberse a que la radiación ionizante comúnmente provoca retraso en la actividad metabólica y la división celular, lo cual retrasa el crecimiento de las plántulas (Chandrashekar 2014, Olasupo et al. 2016).

El comportamiento del RF mostró tendencia cuadrática $(p=0.03)$, no obstante, las únicas dosis que presentaron diferencias $(p<0.01$ ) con el tratamiento testigo fueron 1400 y 2000 Gy (Figura 2a). Cabe señalar que todas las plántulas que emergieron de la dosis de 2000 Gy murieron antes de $21 \mathrm{~d}$ de edad. Debido a esto, la variable RF en este tratamiento fue cero y lo mismo ocurrió para las otras variables evaluadas. De acuerdo con la ecuación de regresión, en esta variable la $R_{50}$ se presentó a 1528.3 Gy. De manera similar, la PS presentó tendencia cuadrática $(p<0.01)$ y las únicas dosis que presentaron diferencias $(p<0.01)$ con el tratamiento testigo fueron 1400 y 2000 Gy (Figura $2 b)$. De acuerdo con la ecuación de regresión y la PS la $\mathrm{RG}_{50}$ se presentó a $1536.2 \mathrm{~Gy}$.

La variable NT mostró tendencia cuadrática $(\mathrm{p}=0.0008)$. El único tratamiento diferente $(\mathrm{p}<$ 0.05) al testigo fue el de 2000 Gy (Figura 2c). En esta variable, la $R_{50}$ se tuvo en $1648.7 \mathrm{~Gy}$. Del mismo modo la AP mostró tendencia cuadrática ( $p$ $<0.0001)$ y el único tratamiento diferente $(\mathrm{p}<$ 0.05) al testigo fue el de 2000 Gy (Figura 2d). De acuerdo con esta variable, la $R_{50}$ se presentó a 1666.9 Gy. El DM presentó tendencia cuadrática ( $p$ $=0.01$ ), en esta variable los tratamientos que presentaron diferencias $(p<0.01)$ con respecto al testigo fueron 1400 y 2000 Gy (Figura 2e). De acuerdo a la ecuación de regresión y el $\mathrm{DM}$, la $\mathrm{RG}_{50}$ se presentó a $1592.6 \mathrm{~Gy}$. El ICC presentó comportamiento cuadrático $(p=0.0008)$. Al igual que en la variable anterior, los tratamiento con diferente ICC ( $p<$ 0.01 ) con respecto al testigo fueron 1400 y 2000 Gy; sin embargo, en la dosis de $1400 \mathrm{~Gy}$, el ICC pre- sentó incremento ( $p<0.01$, Figura $2 f)$. De acuerdo con el ICC la RG 50 se presentó a 1983.5 Gy. La media ponderada de la $D_{50}$ y la $R G_{50}$ resultantes de las nueve variables fue de $1533 \mathrm{~Gy}$.

Las variables RF, PS, NT, AP, DM disminuyeron al incrementar la dosis de radiación. Esto probablemente se debe a que la exposición a radiación gamma con frecuencia causa la destrucción de compuestos de importancia para el desarrollo vegetal, como auxinas y ácido ascórbico (Shah et al. 2008). Además, puede deberse a que la radiación puede causar daños en la estructura del ADN que en la mayor parte de las ocasiones afecta de forma negativa el desarrollo de las plantas (Kiong et al. 2008). Por otro lado, el ICC presentó un incremento con la dosis de $1400 \mathrm{~Gy}$. Lo que concuerda con Jan et al. (2013), quienes encontraron que la radiación puede activar el sistema de los pigmentos fotosintéticos. Lo anterior, confirma que el comportamiento del pasto africano pudo haber sido influenciado por una radio estimulación, por la modificación ocurrida en el ICC. En este sentido, determinar la dosis óptima de radiación ayudará para realizar futuros trabajos en mutagénesis.

En la semilla irradiada a 2000 Gy hubo emergencia de plántulas, no obstante, ninguna logró sobrevivir más de dos semanas. Este fenómeno pudo deberse a que la radiación provocó un desorden metabólico en el pasto africano. Este mismo efecto se reportó por Marcu et al. (2013), quienes encontraron que las plántulas de maíz que emergieron de semilla irradiada con dosis mayores a $500 \mathrm{~Gy}$, no fueron capaces de sobrevivir más de $10 \mathrm{~d}$. La $\mathrm{DL}_{50}$ y las $R_{50}$ en este estudio variaron de 1357 a 2076 Gy. Para inducir variabilidad genética que posibilite la selección y mejoramiento genético de esta especie, se recomienda utilizar la media ponderada de ambos parámetros, la cual se obtuvo a 1533 Gy. Estos resultados permitirán la inducción de nuevo material genético de interés agronómico y ecológico.

\section{AGRADECIMIENTOS}

Al Consejo Nacional de Ciencia y Tecnología (CONACyT). Al Centro Experimental 

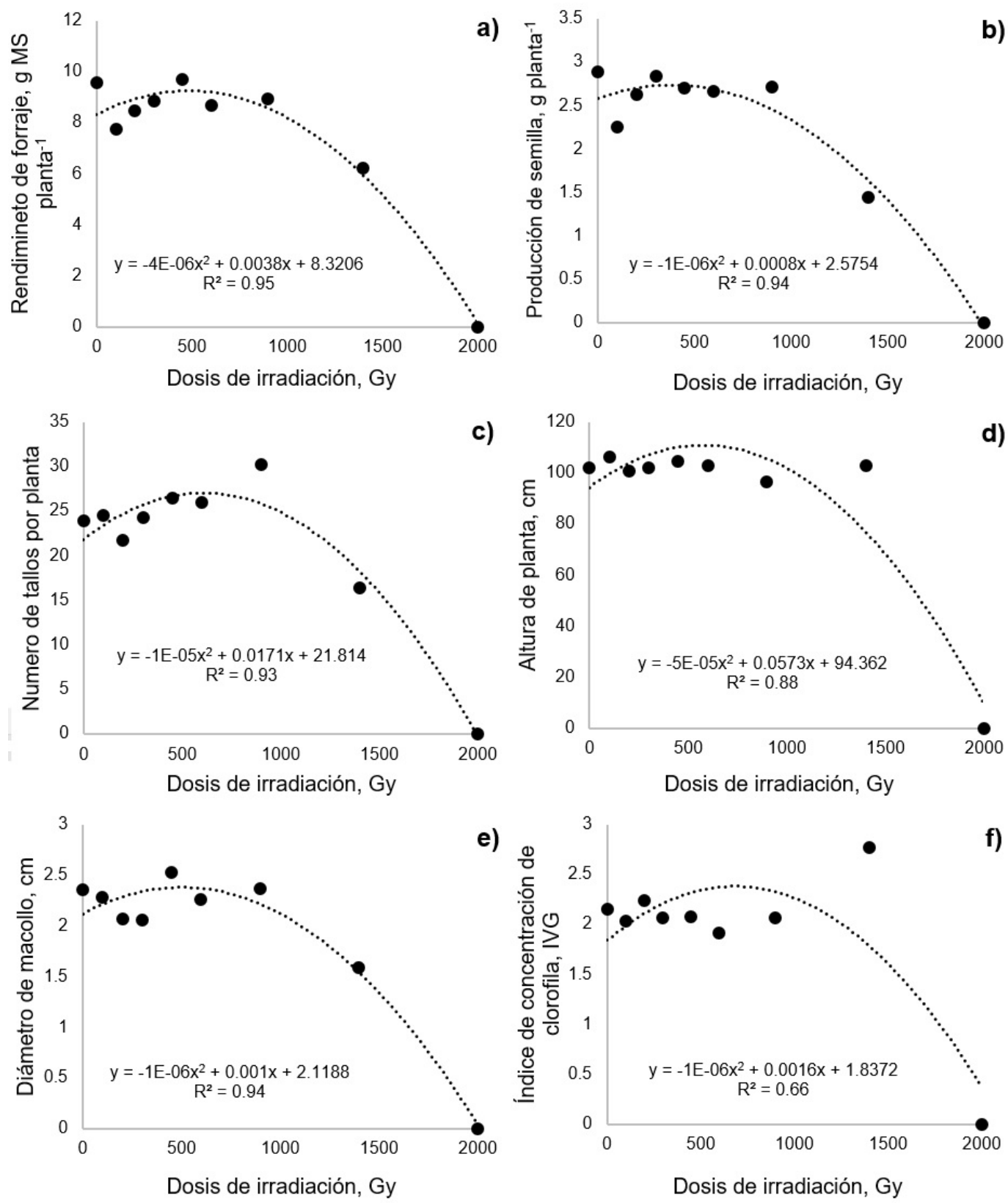

Figura 2. Efecto de diferentes dosis de irradiación con $\mathrm{CO}^{60}$ sobre el rendimiento de forraje (a), producción de semilla (b), número de tallos (c), altura de planta (d), diámetro de macollo (e) e índice de concentración de clorofila $(f)$ en pasto africano (Eragrostis lehmanniana).

Rosario Izapa, Chiapas del INIFAP. Al com-

Domínguez, Chiapas. A la Facultad de Zootecnia y plejo MOSCAMED de SENASICA, Metapa de Ecología de la Universidad Autónoma de Chihuahua.

\section{LITERATURA CITADA}

Akgüm I, Tosun M (2004) Agricultural and cytological characteristics of M1 perennial rye (Secale montanum 
Guss.) as affected by the application of different doses of gamma rays. Pakistan Journal of Biological Sciences 7: 827-833.

Alegre BN (2001) Reacción celular ante la radiación. Radiobiología 1: 9-11.

Ambavane AR, Sawardekar SV, Sawantdesai SA, Gokhale NB (2014) Studies on mutagenic effectiveness and efficiency of gamma rays and its effect on quantitative traits in finger millet (Eleusine coracana $\mathrm{L}$. Gaertn). Journal of Radiation Research and Applied Sciences 8: 120-125.

Ángeles-Espino A, Valencia-Botín AJ, Virgen-Calleros G, Ramírez-Serrano C, Paredes-Gutiérrez L, HurtadoDe la Peña S (2013) Determinación de la dosis letal $\left(\mathrm{DL}_{50}\right)$ con $\mathrm{Co}^{60}$ en vitroplántulas de Agave tequilana var. Azul. Revista Fitotecnia Mexicana 36: 381-386.

Chandrashekar KRA (2014) Gamma sensitivity of forest plants of Western Ghats. Journal of Environmental Radioactivity 132: 100-107.

Flanders A, Kuvlesky W, Ruthven D, Zaiglin R, Bingham R, Fulbright T, Hernández F, Brennan L (2006) Effects of invasive exotic grasses on South Texas rangeland breedeing birds. The Auk 123: 171-182.

Golubinova I, Gecheff K (2011) M1 cytogenetic and physiological effects of gamma-rays in sudan grass (Sorghum Sudanense (Piper.) Stapf). Bulgarian Journal of Agricultural Sciences 17: 417-423.

Harding SS, Johnson SD, Taylor DR, Dixon CA, Turay MY (2012) Effect of gamma rays on seed germination, seedling height, survival percentage and tiller production in some rice varieties cultivated in Sierra Leone. American Journal of Experimental Agriculture 2: 247-255.

Hupy CM, Whitfordb WG, Jacksonc EC (2004) The effect of dominance by an alien grass species, Lehmann lovegrass, Eragrostis lehmanniana, on faunalpedoturbation patterns in North American Desert grasslands. Journal of Arid Environments 58: 321-334.

Jan S, Parween T, Hameed R, Siddiqi TO, Mahmooduzzafar (2013) Effects of presowing gamma irradiation on the photosynthetic pigments, sugar content and carbon gain of Cullen corylifolium (L.) Medik. Chileanjar 74: 345-350.

Khalil SA, Zamir R, Ahmad N (2014) Effect of different propagation techniques and gamma irradiation on major steviol glycoside's content in Stevia rebaudiana. Journal of Animal and Plant Sciences 24: 1743-1751.

Kiong ALP, Lai AG, Hussein S, Harun AR (2008) Physiological responses of Orthosiphon stamineus plantlets to gamma irradiation. American-Eurasian Journal of Sustainable Agriculture 2: 135-149.

Maguire JD (1962) Speed of germination-aid in selection and evaluation for seedling emergence and vigor. Crop Science 2: 176-177.

Marcu D, Damian G, Cosma C, Cristea V (2013) Gamma radiation effects on seed germination, growth and pigment content, and ESR study of induced free radicals in maize (Zea mays). Journal of Biological Physics 39: 625-634.

McGlone MC, Huenneke F (2004) The impact of a prescribed burn on introduced Lehmann lovegrass versus native vegetation in the northern Chihuahuan Desert. Journal of Arid Environments 57: 297-310.

Olasupo FO, Ilori CO, Forster BP, Bado S (2016) Mutagenic Effects of Gamma Radiation on Eight Accessions of Cowpea (Vigna unguiculata [L.] Walp.). American Journal of Plant Sciences 7: 339-351.

O'Reagain PJ, Mentis MT (1989). The effect of plant structure on the acceptability of different grass species to cattle. Journal of the Grassland Society of Southern Africa 6: 163-170. 
Ousmane SD, Elegba W, Danso K (2013) Radio-sensibility of pearl millet (Pennisetum glaucum (L.) R. $\mathrm{Br}$.) and cowpea (Vigna unguiculata (L.) Walp.) seeds germination and seedling growth. International Journal of Innovation and Applied Studies 4: 665-671.

Rajarajan D, Saraswathi R, Sassikumar D (2016) Determination of lethal dose and effect of gamma ray on germination percentage and seedling parameters in ADT (R) 47 rice. International Journal of Advanced Biological Research 6: 328-332.

Ramchander S, Ushakumari R, Pillai MA (2015) Lethal dose fixation and sensitivity of rice varieties to gamma radiation. Indian Journal of Agricultural Research 49: 24-31.

Shah TM, Mirza JI, Haq MA, Atta BM (2008) Radio sensitivity of various chickpea genotypes in M1 generation I-Laboratory studies. Pakistan Journal of Botany 40: 649-665.

Songsri P, Suriharn B, Sanitchon J, Srisawangwong S, Kesmala T (2011) Effects of gamma radiation on germination and growth characteristics of physic nut (Jatropha curcas L.). Journal of Biological Sciences 11: 268-274.

Statistical Analysis System (SAS). Institute. SAS/STAT 9.1.3 (2006) User's guide. Cary, NC, USA. 1686p.

Talebi AB, Talebi AB (2012) Radiosensitivity Study for Identifying the Lethal Dose in MR219 (Oryza sativa L. spp. Indica CV. MR219). International Journal of Agricultural Science, Research and Technology 2: 63-67.

Thole V, Peraldi A, Worland B, Nicholson P, Doonan JH, Vain P (2011) T-DNA mutagenesis in Brachypodium distachyon. Journal of Experimental Botany 10: 1-10. 